\title{
Porträt
}

\section{Umsetzung der SDGs in deutschen Städten am Beispiel der Fairtrade-Kampagnen}

Die Sustainable Development Goals (SDGs), 2015 von den Vereinten Nationen verabschiedet, enthalten Verpflichtungen u. a. zu Ernährungssicherheit, nachhaltiger Landwirtschaft, Gleichberechtigung und Recht auf Schulbildung - Bereiche, in denen Fairtrade seit über 25 Jahren tätig ist und Wirkung entfaltet. Auf nationaler Ebene trägt TransFair e.V. (Fairtrade Deutschland) zur Umsetzung der Nachhaltigkeitsziele bei und verankert diese über Bildungs- und Kampagnenarbeit in der Gesellschaft - von der Hauptstadt Berlin mit über 3 Millionen bis zur Hallig Hooge mit seinen 92 Einwohner/-innen.

Was ist Fairtrade? Fairtrade ist eine internationale Bewegung und weltweit bekannt für das Fairtrade-Siegel. 1,7 Mio. Menschen in Ländern des globalen Südens profitieren vom fairen Handel: von stabilen Preisen, einer zusätzlichen Fairtrade-Prämie, den Standards, die z. B. Arbeitsschutzkriterien, Verbot von Kinderarbeit und Förderung von nachhaltiger Anbaupraxis umfassen. TransFair e.V. repräsentiert Fairtrade in Deutschland. Der 1992 gegründete gemeinnützige Verein wird von 35 Mitgliedsorganisationen der (kirchlichen) Entwicklungszusammenarbeit, Bildung, Umwelt und Politik getragen. TransFair fördert fairen Konsum und schafft Absatzmöglichkeiten für Fairtrade-Produkte. Darüber hinaus verfolgt der Verein einen klaren Bildungsauftrag: das Bewusstsein für nachhaltigen Konsum zu fördern und Verbraucher/-innen sowie Wirtschaft und Politik zu fairem Konsum zu motivieren. Die globale Fairtrade-Bewegung und TransFair als Organisation für fairen Handel in Deutschland, leisten einen konkreten Beitrag, die UN-Ziele umzusetzen. SDG 12 mit seinem Schwerpunkt auf nachhaltigen Produktions- und Konsummustern hat dabei besonders hohe Relevanz.

Mit Kampagnen Menschen vor Ort motivieren: Menschen zusammenbringen, die sich gemeinsam für fairen Handel einsetzen - das ist die Idee hinter den internationalen Fairtrade-Kampagnen. Diese bieten konkrete Handlungsoptionen und einen Fahrplan zur Umsetzung der SDGs sowie zur Implementierung globaler Nachhaltigkeitsstrategien auf lokaler Ebene. Bereits 2009 rief TransFair die Kampagne Fairtrade-Towns in Deutschland ins Leben. Das Interesse ist seit dem Gründungsjahr kontinuierlich gewachsen: Im Herbst 2020 erhielt die 700. Kommune den Titel. Inzwischen leben über die Hälfte der Menschen in Deutschland in einer Fairtrade-Town. Mit den Schwesterkampagnen FairtradeSchools (seit 2012) und Fairtrade-Universities (seit 2014) werden zudem gezielt junge Menschen angesprochen. Über 755 Schulen und 32 Universitäten sind (Stand November 2020) ausgezeichnet. Um als Fairtrade-Kommune, -Schule oder -Hochschule ausgezeichnet zu werden, müssen fünf Kriterien erfüllt sein, die zur Umsetzung der SDGs beitragen: Ein offizieller Beschluss formalisieren die Aktivitäten zu mehr fairem Handel, im Einzelhandel und in der Gastronomie werden fair gehandelte Produkte angeboten. Eine Steuerungsgruppe ein Schul- oder Hochschulteam koordiniert die Aktivitäten der
Stadt bzw. der (Hoch-)Schule zum fairen Handel, organisiert Bildungsprojekte, Veranstaltungen und Aktionen und kümmert sich um Öffentlichkeitsarbeit. Die Vernetzung in Fairtrade-Towns trägt dazu bei, die Beschaffung von Konsumgütern fairer zu gestalten. Die Umstellung beginnt zumeist mit fairem Kaffee oder Tee in Rathäusern und wird dann häufig kontinuierlich ausgebaut, z. B. auf Fairtrade-Blumen oder Präsentkörbe als Geschenke oder gar auf die Ausstattung der städtischen Betriebe: Die Stadt Würzburg etwa beschafft die Berufsbekleidung der Stadtreinigung nach fairen Kriterien.

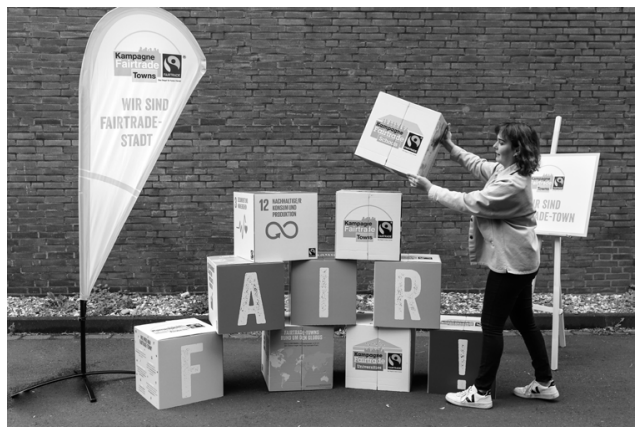

Abb. 1: Von New York bis Hallig Hooge - Fairtrade Towns, Quelle: Fairtrade Deutschland

Fairer Handel als Baustein von Bildung für Nachhaltige Entwicklung: Die Kampagnen setzen aber auch entwicklungspolitische Bildungsarbeit als einen wichtigen Baustein um. An Fairtrade-Schools und -Universities ist Nachhaltigkeit stets präsent - indem fairer Handel im Unterricht und als Lehrthema behandelt wird und die Fairtrade-(Hoch-)Schulteams regelmäßige Aktionen dazu organisieren. Durch ihre aktive Mitwirkung erleben Schüler/-innen und Studierende, dass sie die eigenen Institutionen aber auch das eigene private Umfeld fairer und nachhaltiger gestalten können. Ein Best-PracticeBeispiel kommt aus der Universität zu Köln, eine von derzeit 31 Fairtrade-Universities: Im August 2020 wurde hier eine „Kompetenzstelle Fairer Handel“ eingerichtet, welche die Lehre und Forschung zum fairen Handel an der Universität zu Köln bündelt und weiter ausbaut. Globale Nachhaltigkeitsstrategien werden durch die Kampagnen wie Fairtrade-Towns, -Schools und -Universities in die jeweiligen gesellschaftlichen Gruppen getragen und knüpfen dort an die Arbeits- und Wirkungsbereiche an, in denen die Zielgruppen selbst aktiv werden können. Engagierte bekommen konkrete Handlungsoptionen, werden bei ihrer Umsetzung unterstützt und setzen so globale Vorgaben lokal um - auf beschaulichem Hallig Hooge wie im großen Berlin. Weitere Informationen zu den Kampagnen können unter www.fairtrade-kampagnen.de abgerufen werden.

Edith Gmeiner, Lisa Herrmann \& Kristina Klecko Fairtrade Deutschland doi.org/10.31244/zep.2020.04.06 